\title{
ACOUSTIC EMISSION DIAGNOSIS SYSTEM AND WIRELESS MONITORING FOR DAMAGE ASSESSMENT OF CONCRETE STRUCTURES
}

\author{
Dong-Jin Yoon, Sang-Il Lee, Chi-Yeop Kim and Dae-Cheol Seo
}

Korea Research Institute of Standards and Science, Korea

\begin{abstract}
Concrete is the most widely used materials for building civil structures. In the nondestructive evaluation point of view, a lot of acoustic emission (AE) signals are generated in concrete structures under loading whether the crack is active or not. First, this study aims to identify the differences of AE event patterns caused by both actual crack development and the other sources. Secondly, it was focused to develop acoustic emission diagnosis system for assessing the deterioration of concrete structures such as a bridge, dame, building slab, tunnel etc.. Thirdly, a feasible study was done for the application of wireless acoustic emission sensor module to concrete structures. From the previous laboratory study such as AE event patterns analysis under various loading conditions, we confirmed that AE analysis provided a promising approach for estimating the condition of damage and distress in concrete structures. In this work, the algorithm for determining the damage status of concrete structures was developed and some criteria for decision making were also suggested. For the future application of wireless monitoring, a compact wireless acoustic emission sensor module was developed and applied to the concrete beam for performance test. Finally, based on the selfdeveloped diagnosis algorithm, new AE system for practical AE diagnosis was demonstrated for assessing the conditions of damage and distress in concrete structures.
\end{abstract}

Keywords: Acoustic emission, Diagnosis system, Concrete structures, Damage assessment, Wireless monitoring

\section{INTRODUCTION}

In comparison with other non-destructive techniques, Acoustic Emission (AE) technique has two important advantages: AE technique can give some information about defects inside of the material, and it is capable of real time monitoring. So, the AE technique has emerged as a powerful non-destructive tool to detect or evaluate damages in the field of safety of civil structures. Previous AE research on concrete have been focused on characterizing the failure mechanisms in cement-based materials and on evaluating integrity of concrete structures [1-3]. However, the application of $\mathrm{AE}$ technique to evaluate the integrity of concrete safety is not commonly used yet, because the AE technique requires some expert skills in data acquisition, signal process, and estimation. Therefore, a systematic approach is needed to assess and monitor the deterioration of concrete structure using AE technique. The objective of this study is to develop a new algorithm for assessing the integrity of concrete structures using $\mathrm{AE}$ 
technique.

\section{ACOUSTIC EMISSION SOURCES IN REINFORCED CONCRETE BEAM}

Generally, AE sources in concrete are known by three causes: crack development, friction between concrete and reinforcement, and break of fibers in fiber reinforced concrete [4]. To apply $\mathrm{AE}$ technique for evaluating the integrity of concrete structure, $\mathrm{AE}$ sources caused from cracks need to be subdivided into development of micro cracks, flexural cracks, diagonal tension cracks, and friction of existing crack surfaces. Fig. 1 shows a result of AE source location in RC beam [5]. This classification is important because some kinds of cracks were essential and allowed in RC beams.
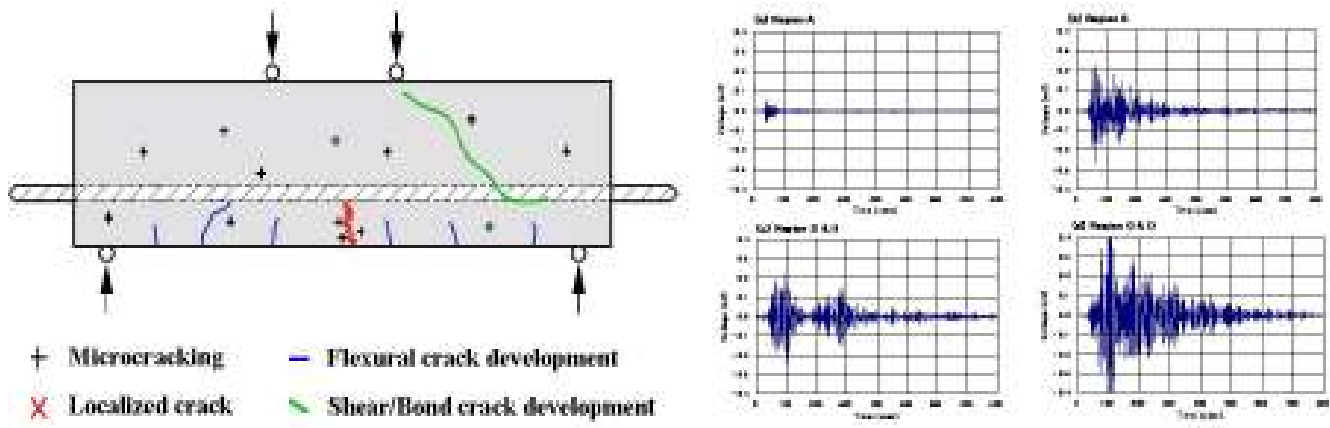

Fig. 1 AE signals in concrete beam

As shown in Fig. 2, contrary to concrete beam without reinforcement, RC beams have unique AE behavior called secondary peak [6] which is generation of AE hits during unloading. The secondary peak is presumed to be caused by friction while flexural cracks are closing, and it is expected that the ratio of primary and secondary peak would be useful for evaluating integrity. In most of the previous studies, AE signals caused by friction were considered as noise although it is hard to separate from the signals caused by crack propagation [7]. In this study, the signals from the friction of crack surfaces were considered as normal signals, and the differences between normal and abnormal signals were identified through a series of experiments 

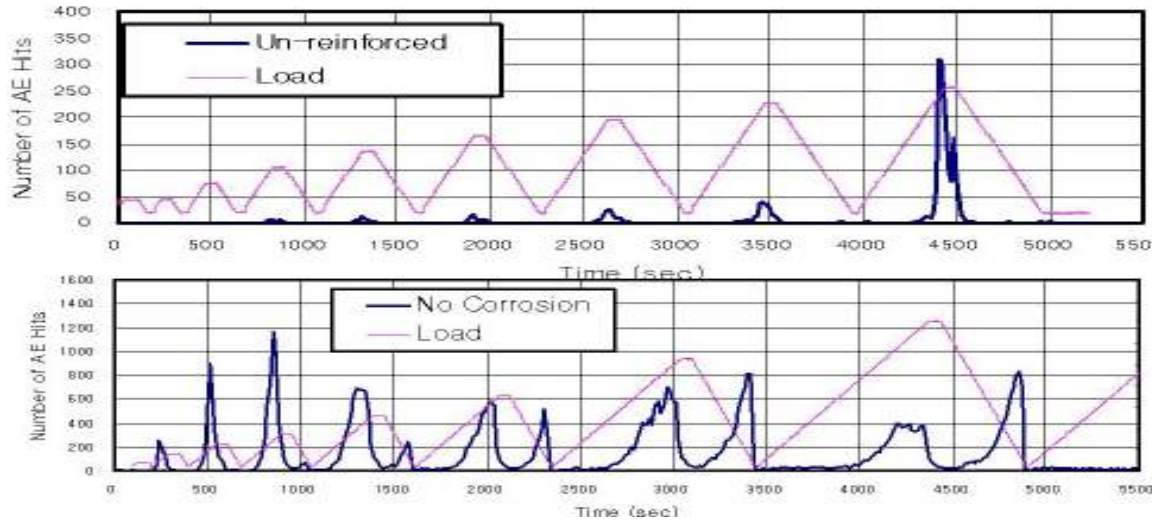

Fig. 2 Secondary peak of AE in RC beam

\section{EXPERIMENTS}

A series of four points bending tests for two types of reinforced concrete beam specimens was carried out to obtain $\mathrm{AE}$ event patterns under various loading conditions. To confirm the repeatability of the test results, the same series of loading tests were repeated for three specimens for each type of beam. Fig. 3 (a) shows the drawings of reinforced concrete beam used for the tests. Multi-channel commercial AE system, MISTRAS (PAC) was used to acquire AE signals. Three $150 \mathrm{kHz}$ resonant type AE sensors (R15) were used with the sensor arrangement shown in Fig. 3 (b). Ch. 2 was monitored signals from flexural cracks and Ch. 1 and 3 were monitored signals from diagonal cracks. $500 \mathrm{kN}$ hydraulic actuator (MTS) was used to apply loads.

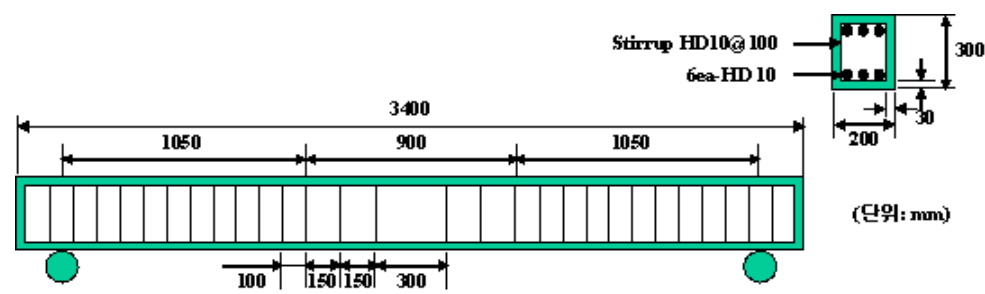

(a) beam specification

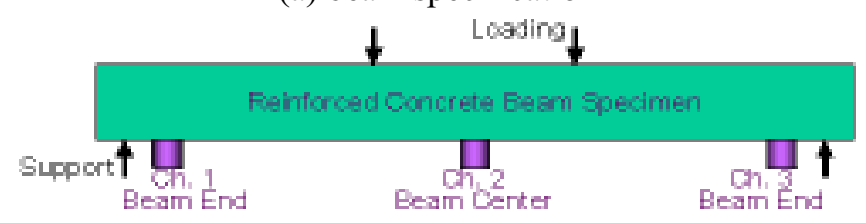

(b) AE sensor location

Fig. 3 Concrete beam specimen and sensor location

In this experiment, typical 4-point bending test was applied to all specimens. As a loading procedure, a stepwise cyclic loading test was carried out during first stage and then failure test was done to rupture of concrete beam by displacement control. Fig. 4 shows the load profile of 10-stepwise-cycles and time-displacement curve. The velocity of loading and unloading in 
the cyclic loading test was $10 \mathrm{kN} / \mathrm{min}$ and holding time was set to 3 min. Fig. 5 shows the AE sensors attached on the concrete beam upper surface and the jig for 4-point bending.
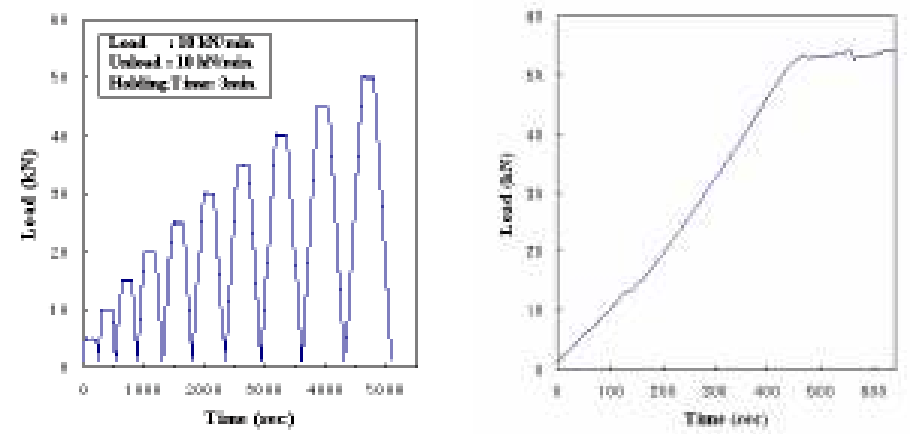

Fig. 4 Load profiles for the cyclic load test and failure test

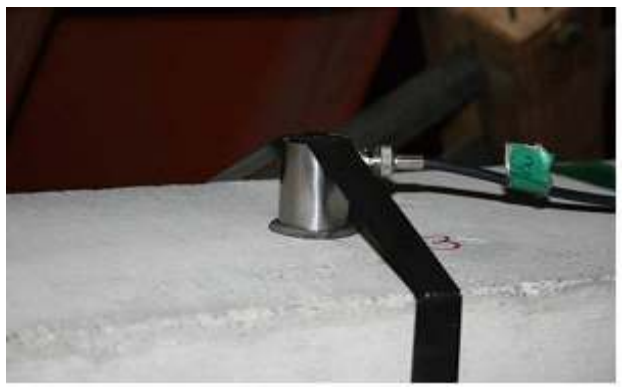

(a) sensor on the concrete

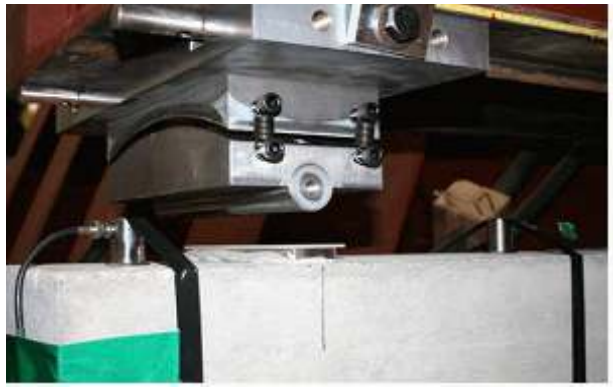

(b) specially-designed jig

Fig. 5 Attached AE sensor and jig for 4-point bending test

\section{AE ACTIVITIES ACCORDING TO DAMAGES PATTERNS}

Fig. 6 compares typical AE hits and amplitude during 10-stepwise-loading-cycle for each channel. Differences in the number of overall $\mathrm{AE}$ hits and ratio of primary and secondary hits are obvious. Also, the hit occurrence at $\mathrm{CH}-2$ (center of beam) was higher than the other two channels $\mathrm{CH}-2$ and $\mathrm{CH}-3$ (side of beam). This means that most of crack was occurred at the center of beam, especially at tension side of the beam surface. This phenomenon can be used to verify the failure of beam in the left or right hand side by comparing the occurrence of $\mathrm{AE}$ hit in each channel.

As shown in Fig. 6(a), it was found that the activity of secondary peak for each cycle was totally different between $\mathrm{CH} \_2$ and $\mathrm{CH}-1$ or $\mathrm{CH}-3$. And it was also found that the difference of AE primary and secondary hit occurrence was very distinct between in the early stages and later stages of cycle as shown in Fig. 7. 


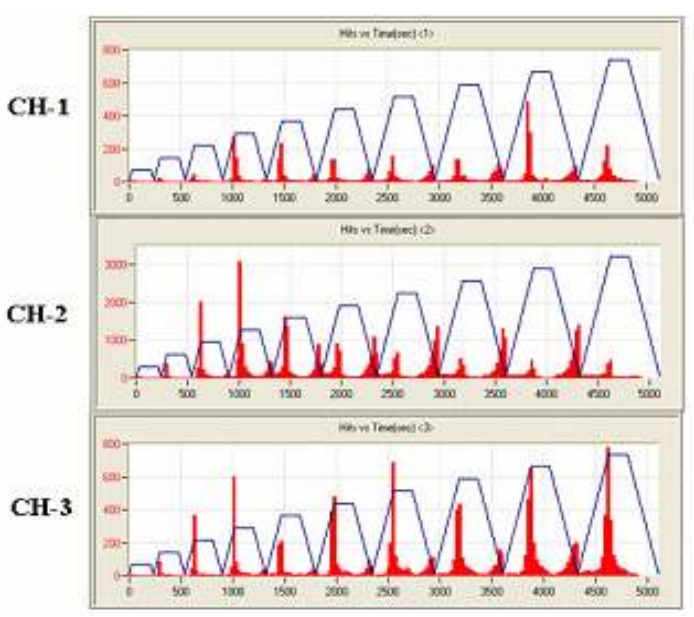

(a) AE hit

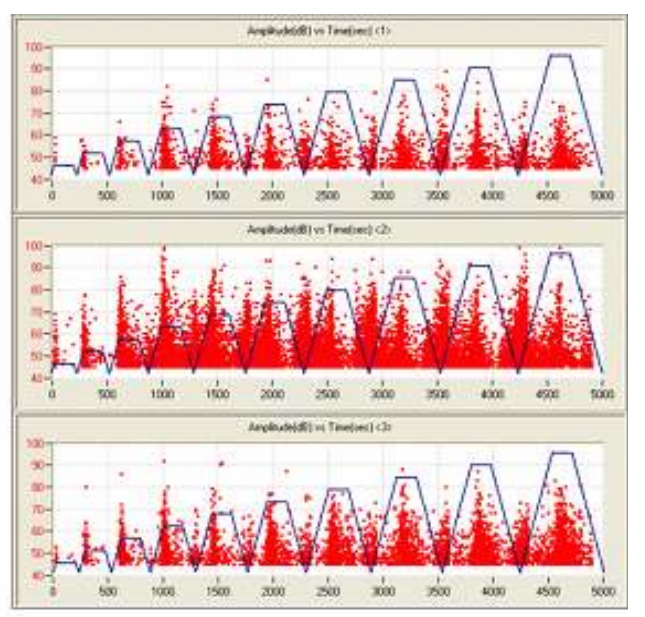

(b) amplitude

Fig. $6 \mathrm{AE}$ hit and amplitude as a function of elapsed time

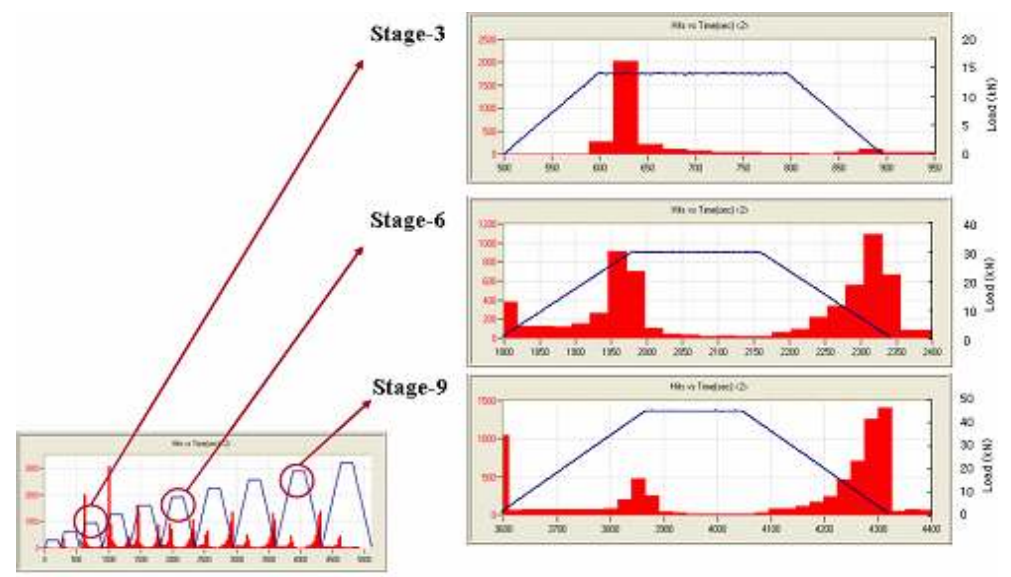

Fig. 7 Comparison of AE hit occurrence coming form three stages

\section{AE KEY PARAMETER ANALYSIS FOR DAMAGE ASSESSMENT}

Fig. 8 shows a process for applying AE key parameter to damage assessment and criteria for real-time health monitoring for concrete structure. This evaluation procedure will be processed by three steps such as $\mathrm{AE}$ signal acquisition, $\mathrm{AE}$ parameter extraction, risk based decision through overall evaluation of AE key parameter. 


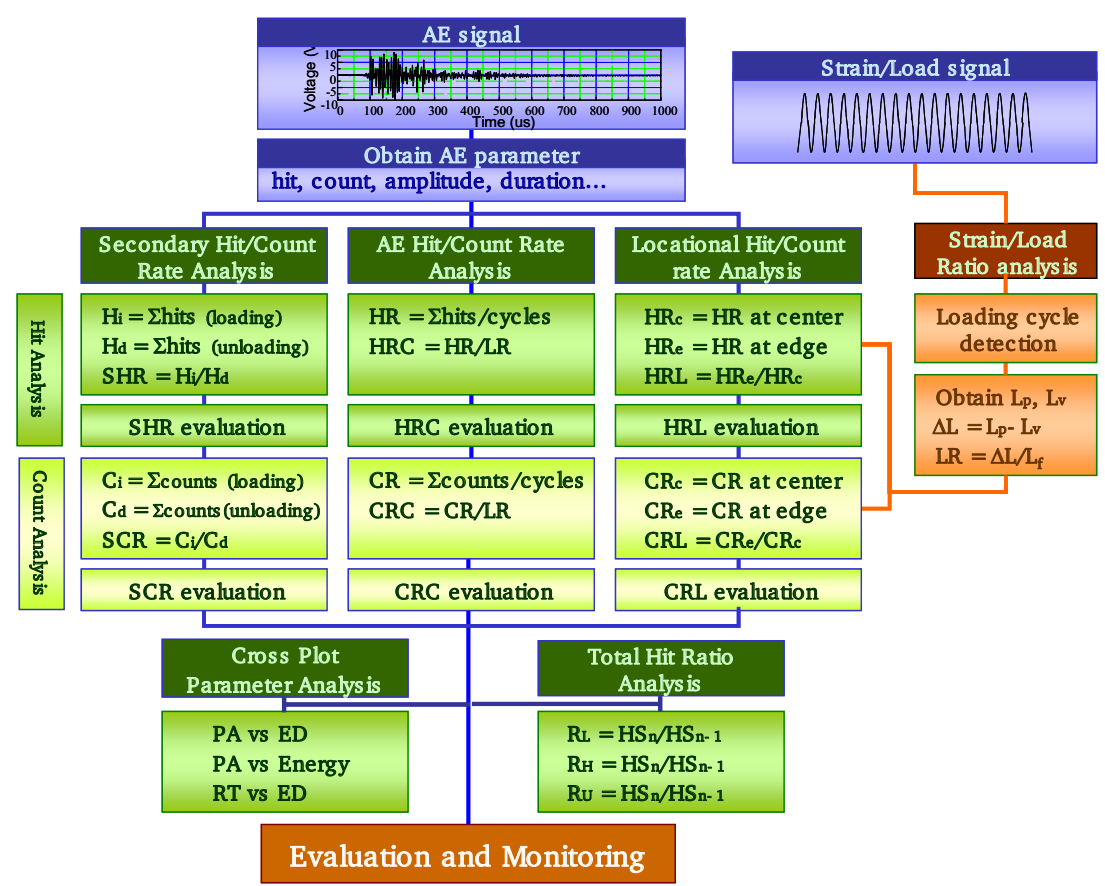

Fig. 8 Process of AE key parameter analysis

\section{DESIGN AND FABRICATION OF INTEGRATED AE WIRELESS SENSOR}

The wireless acoustic emission was developed in the type of whole integration which composed of sensing piezo-element, pre-amplification module, analog/digital circuit, wireless module, and power management circuit. Based on the ATmega 128 processor, control program of sensor was installed. The bandwidth of frequency was about $50 \mathrm{kHz}-300 \mathrm{kHz}$ and $\mathrm{AE}$ signal can be amplified to maximum $60 \mathrm{~dB}$. Simple AE parameters such as hit, count, duration, time etc. were measured and displayed. Wireless transmit of measured data was carried by $2.4 \mathrm{GHz}$ Zigbee(IEEE802.15.4 Protocol) module. Inside battery of 3.6V Lithium-ion cell is able to use 0.7 voltage with $94 \%$ efficiency by power circuit. Fig. 9 shows the developed prototype AE wireless sensor module. Fig. 10 shows also the block diagram for the wireless sensor module.
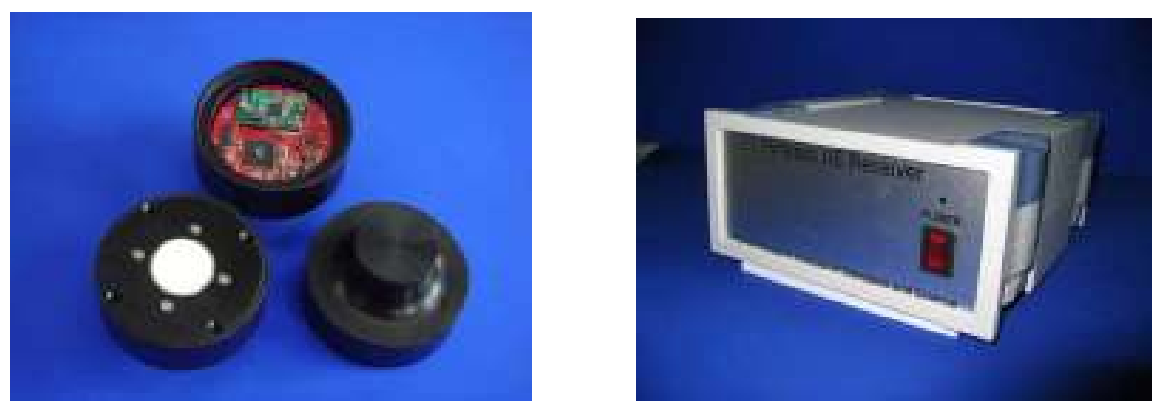

Fig. 9 Wireless AE sensor module and receiver 


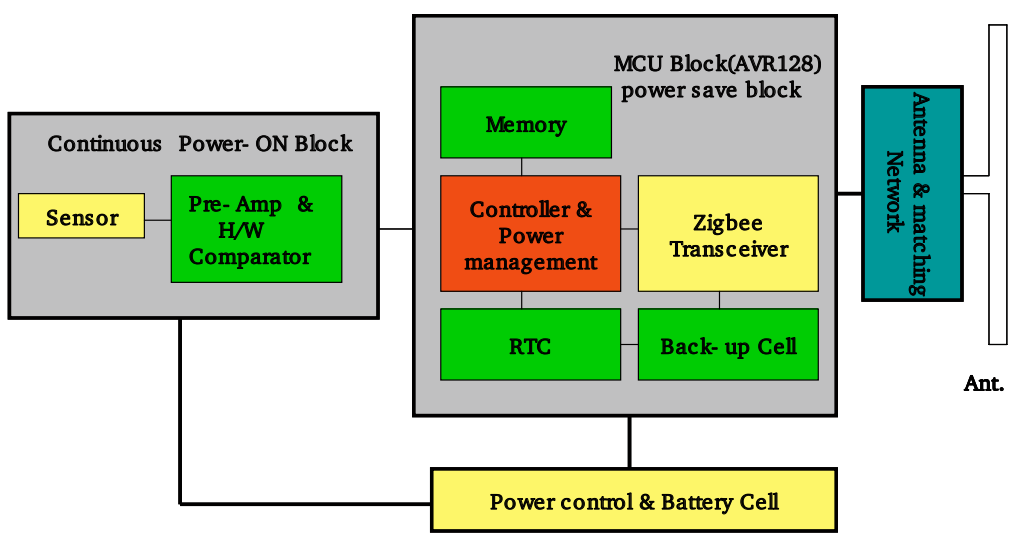

Fig. 10 Wireless AE sensor Block-Diagram

\section{TEST AND VERIFICATION OF DEVELOPED SENSOR}

Channel 1 of Fig. 11 indicates the typical AE sensor signal by pencil lead break on the concrete beam surface. Channel 2 also shows the result of wave shaping by comparator. Channel 3 shows the output of ripple counter calculated by twice variation of input pulse. Finally, the output of AE signals was transmitted to $\mathrm{AE}$ receiver module and then it was shown in the main display of wireless AE monitoring system as shown in Fig. 12.

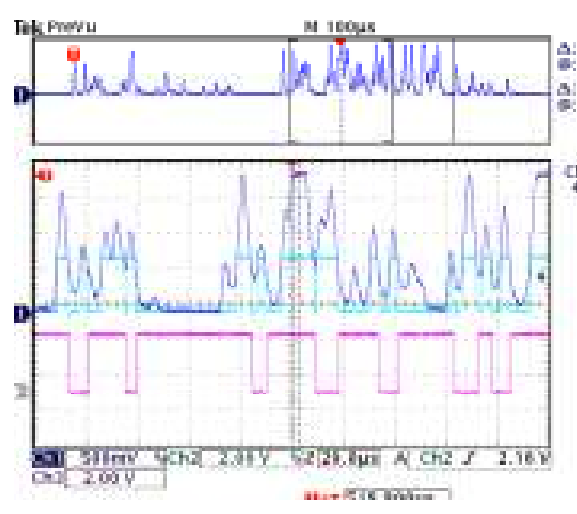

[AE Pre amp out wave form]

CH1: AE sensor pre- amp Out waveform
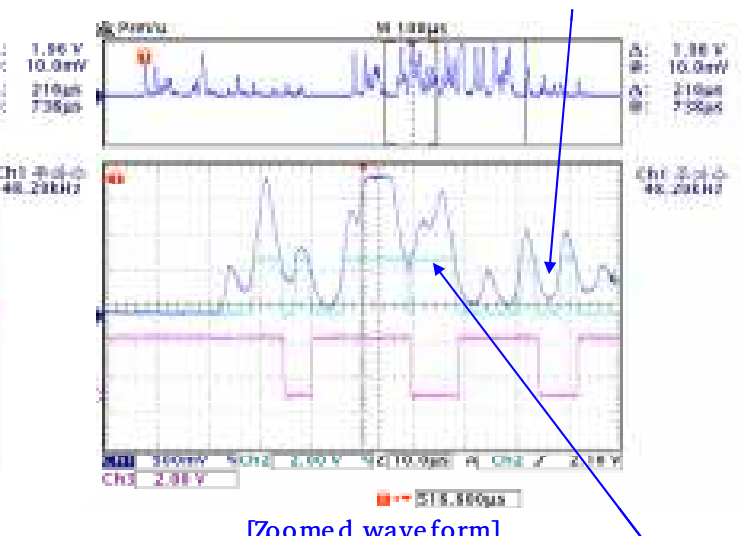

[Zoomed wave form]

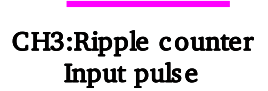

Trigger level $=700 \mathrm{mV}$ (adjus table)

Fig. 11 Waveform of sensor output, trigger signal \& count pulse 


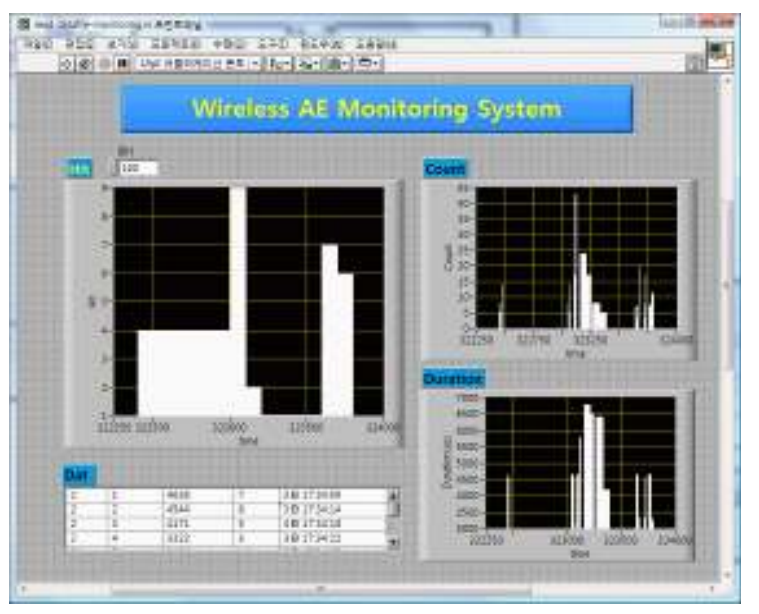

Fig. 12 The main display of wireless AE monitoring system

\section{CONCLUDING REMARK}

This study was aimed at developing a new method for assessing integrity of concrete structures and also at verifying a developed wireless AE monitoring module to concrete beam specimen. In this work, the algorithm for determining the damage status of concrete structures was developed and some criteria for decision making were also suggested. For the future application of wireless monitoring, a compact wireless acoustic emission sensor module was developed and applied to the concrete beam for performance test. Finally, based on the selfdeveloped diagnosis algorithm, new AE system for practical AE diagnosis was demonstrated for assessing the conditions of damage and distress in concrete structures.

\section{REFERENCES}

[1] C. Ouyang, E. Landis, and S. P. Shah, "Damage assessment in concrete using quantitative acoustic emission", Journal of Engineering Mechanics, Vol. 117, No. 11, 1991, pp. 2681-2698. [2] S. P. Shah, S. E. Swartz, and C. Ouyang, Fracture Mechanics of Concrete: Application of Fracture Mechanics to Concrete, Rock and Other Quasi-Brittle Materials, John Wiley \& Sons Inc., NY, 1995.

[3] S. Yuyama, T. Okamoto, and S. Nagataki, "Acoustic emission evaluation of structural integrity in repaired reinforced concrete beams", Material Evaluation, Jan., 1994, pp. 86-90.

[4] Sidney Mindess, "Acoustic Emission Method", CRC Handbook on Nondestructive Testing of Concrete, CRC Press, 1991, p. 317.

[5] D. J. Yoon, W. J. Weiss, and S. P. Shah, "Detecting the extent of corrosion with acoustic emission", Transportation Research Record, No. 1698, 2000, pp. 54-60.

[6] D. J. Yoon, W. J. Weiss, and S. P. Shah, "Assessing damage in corroded reinforced concrete using acoustic emission", Journal of Engineering Mechanics, ASCE, Vol. 126, No. 3, 2000, pp. 273-283.

[7] E. G. Nesvijski, "Failure forecast and the acoustic emission 'Silence Effect' in concrete", Proc. of ASNT Spring Meeting, 1996, pp. 108-110. 